\title{
Electrostatic self-energies of discrete charge distributions on Jordan curves
}

\author{
Jerzy Cioslowski · Joanna Albin
}

Received: 7 July 2014 / Accepted: 19 July 2014 / Published online: 5 August 2014

(C) The Author(s) 2014. This article is published with open access at Springerlink.com

\begin{abstract}
The characteristic energy of a smooth Jordan curve of length $L$, defined as the coefficient in the term proportional to $N^{2} / L$ in the large- $N$ asymptotics of the minimal electrostatic self-energy of $N$ unit charges located on the curve in question, possesses an expansion involving the function $\varphi(t)$ that measures the deviation from linearity in the dependence of the tangential angle on the arc length. The leading term in this expansion is given by a functional that is quadratic in $\varphi(t)$. The explicit expression for this functional can be derived without taking into account the energy lowering due to relaxation of the particle positions that, being of the order of $N^{2}(\ln N)^{-1}$ for large $N$, does not contribute to the characteristic energy.
\end{abstract}

Keywords Jordan curves · Electrostatic self-energy $\cdot$ Discrete charge distributions

\section{Introduction}

Confinement of interacting classical particles gives rise to diverse patterns of particle positions at equilibrium geometries. The combination of Coulombic interparticle interactions and two-dimensional confining potentials of cylindrical symmetry usually produces assemblies of particles positioned on either vertices of polygons inscribed on concentric rings or nodes of triangular lattice [1-15]. Formation of such patterns, which is observed both in experimental settings and numerical simulations, occurs in systems ranging from electrons in quantum dots [2-7] to ions in dusty plasmas [8-10] and triboelectrically charged objects [11].

\footnotetext{
J. Cioslowski $(\varangle) \cdot$ J. Albin

Institute of Physics, University of Szczecin, Wielkopolska 15,

70-451 Szczecin, Poland

e-mail: jerzy@wmf.univ.szczecin.pl
} 
Both the energies and equilibrium geometries of the aforedescribed species are accurately predicted by approximate shell models [16] with well-understood mathematical properties [17]. A generalization of those models to confining potentials lacking cylindrical symmetry would require derivation of an expression for the electrostatic self-energy of a discrete charge distribution located on an equipotential line, which is a Jordan curve. A survey of the relevant literature reveals a paucity of studies on this subject, the seminal work of Martínez-Finkelshtein et al. [18] being the notable exception. In the present paper, we report on research intended to bridge this undesirable gap in knowledge.

The organization of paper is as follows: first, the characteristic energy of a Jordan curve is defined as the coefficient multiplying the ratio $N^{2} / L$, where $N$ is the number of particles and $L$ is the curve length, in the second leading term of the $N \rightarrow \infty$ energy asymptotics (the first term being the universal expression derived in ref. [18]). Second, the perturbative expression for the characteristic energy is derived for curves slightly deviating from circles. Third, relaxation of the particle positions from the Martínez-Finkelshtein minimizer is demonstrated to yield a vanishing contribution to the characteristic energy. Finally, the validity of the derived expressions is demonstrated for a family of simple test curves.

\section{Theory}

Consider a set $\Omega$ of $N$ unit point charges located on a smooth Jordan curve $\Xi \equiv$ $\{x(s), y(s)\}$ conveniently defined by its Whewell representation [19], i.e.

$$
x(s)=x_{0}+\int_{0}^{s} \cos 2 \pi \phi(t / L) d t, \quad y(s)=y_{0}+\int_{0}^{s} \sin 2 \pi \phi(t / L) d t .
$$

It follows from Eq. (1) that the curvature of $\Xi$ is given by

$$
K(s)=\frac{x^{\prime}(s) y^{\prime \prime}(s)-y^{\prime}(s) x^{\prime \prime}(s)}{\left[x^{\prime}(s)^{2}+y^{\prime}(s)^{2}\right]^{3 / 2}}=(2 \pi / L) \phi^{\prime}(s / L) .
$$

Consequently, the corresponding tangential angle equals [19]

$$
\int_{0}^{s} K(t) d t+2 \pi \phi(0)=2 \pi \phi(s / L)
$$

which implies

$$
\underset{k \in \mathbb{Z}}{\forall} \phi(t+k)=k+\phi(t) .
$$

The function

$$
\varphi(t)=\phi(t)-t
$$


measures deviation of $\Xi$ from a circle. It follows from Eq. (4) that $\varphi(t)$ is a periodic function, namely

$$
\underset{k \in \mathbb{Z}}{\forall} \varphi(t+k)=\varphi(t)
$$

The necessary and sufficient conditions for closure of $\Xi$ read

$$
\int_{0}^{1} \cos 2 \pi \phi(t) d t=\int_{0}^{1} \sin 2 \pi \phi(t) d t=0 .
$$

In general, these conditions imply that the period of $\varphi(t)$ is a reciprocal of a natural number greater than one [20].

The electrostatic self-energy $E_{\Xi}(N)$ of $\Omega$ (i.e. its minimal discrete Riesz energy) is given by

$$
E_{\Xi}(N)=(1 / 2) \min _{\left\{s_{k}\right\}} \sum_{k \neq l=1}^{N}\left[d\left(s_{k}, s_{l}\right)\right]^{-1}
$$

where

$$
\begin{aligned}
d\left(s_{k}, s_{l}\right) & =\left(\left[\int_{s_{k}}^{s_{l}} \cos 2 \pi \phi(t / L) d t\right]^{2}+\left[\int_{s_{k}}^{s_{l}} \sin 2 \pi \phi(t / L) d t\right]^{2}\right)^{1 / 2} \\
& =\left(\int_{s_{k}}^{s_{l}} \int_{s_{k}}^{s_{l}} \cos 2 \pi\left[\phi(t / L)-\phi\left(t^{\prime} / L\right)\right] d t d t^{\prime}\right)^{1 / 2} .
\end{aligned}
$$

According to the Martínez-Finkelshtein theorem [18], the large- $N$ asymptotics of $E_{\Xi}(N)$ reads

$$
\lim _{N \rightarrow \infty} \frac{E_{\Xi}(N)}{N^{2} \ln N}=L^{-1}
$$

the corresponding minimizer $\left\{s_{k}\right\}$ in the RHS of Eq. (8) possessing the property

$$
\underset{1 \leq k \leq N-1}{\forall} s_{k+1}-s_{k}=N^{-1} L .
$$

Let the characteristic energy $C_{\Xi}$ of $\Xi$ be defined as

$$
C_{\Xi}=\lim _{N \rightarrow \infty}\left(N^{-2} E_{\Xi}(N) L-\ln N\right) .
$$

The characteristic energy of a circle [21], 


$$
C_{\text {circle }}=\gamma+\ln \frac{2}{\pi} \approx 0.125633
$$

where $\gamma$ is the Euler-Mascheroni constant, enters the LDA (local density approximation) expression

$$
e_{\text {coh }} \approx-2^{5 / 2} \exp \left[-\left(1+\frac{C_{\text {circle }}}{2}\right)\right] \int[\rho(\vec{r})]^{3 / 2} d \vec{r}
$$

for the cohesive energy $e_{c o h}$ of a Coulombic system confined by a radially symmetric potential in two dimensions [17]. It is unclear at present how this expression can be generalized to less symmetrical species. Understanding the properties of $C_{\Xi}$ constitutes the first step towards elucidation of this problem.

For small deviations of $\Xi$ from a circle, the reciprocal of the distance $d\left(s_{k}, s_{l}\right)$ can be expressed in terms of a power series in $\varphi(t)$,

$$
\begin{aligned}
{\left[d\left(s_{k}, s_{l}\right)\right]^{-1}=} & \pi L^{-1} \sin ^{-1} \frac{\pi\left|s_{l}-s_{k}\right|}{L} \\
& +\pi^{3} L^{-2} \sin ^{-2} \frac{\pi\left(s_{l}-s_{k}\right)}{L} \int_{-\left|s_{l}-s_{k}\right|}^{\left|s_{l}-s_{k}\right|} \varphi\left(\frac{s_{k}+s_{l}+t}{2 L}\right) \sin \frac{\pi t}{L} d t \\
& +\pi^{4} L^{-2} \sin ^{-2} \frac{\pi\left(s_{l}-s_{k}\right)}{L} \int_{-\left|s_{l}-s_{k}\right|}^{\left|s_{l}-s_{k}\right|}\left[\varphi\left(\frac{s_{k}+s_{l}+t}{2 L}\right)\right]^{2} \cos \frac{\pi t}{L} d t \\
& +\left(\pi^{5} / 2\right) L^{-3} \sin ^{-3} \frac{\pi\left|s_{l}-s_{k}\right|}{L} \\
& \times\left[2\left[\int_{-\left(s_{l}-s_{k}\right)}^{2} \varphi\left(\frac{s_{k}+s_{l}+t}{2 L}\right) \sin \frac{\pi t}{L} d t\right]^{2}\right. \\
& -\left[\int_{-\left(s_{l}-s_{k}\right)}^{s_{l}-s_{k}} \varphi\left(\frac{s_{k}+s_{l}+t}{2 L}\right) \cos \frac{\pi t}{L} d t\right]^{2}+\cdots
\end{aligned}
$$

The only term that diverges as $s_{l}$ approaches $s_{k}$ is that independent of $\varphi(t)$, which explains the universality of the asymptotics (10). On the other hand, all the remaining terms potentially contribute to $C_{\Xi}$.

The contribution to $C_{\Xi}$ linear in $\varphi(t)$, computed at the minimizer (11), is given by

$$
C_{\Xi}^{(1)}=\lim _{N \rightarrow \infty}\left(\pi^{3} / 2\right) N^{-2} L^{-1} \sum_{k \neq l=1}^{N} \sin ^{-2} \frac{\pi(l-k)}{N}
$$




$$
\begin{aligned}
& \times \int_{-(L / N)|l-k|}^{(L / N)|l-k|} \varphi\left(\frac{k+l}{2 N}+\frac{t}{2 L}\right) \sin \frac{\pi t}{L} d t \\
= & \lim _{N \rightarrow \infty}\left(\pi^{3} / 2\right) N^{-2} L^{-1} \sum_{k=1}^{N} \sum_{j=1}^{N-1} \sin ^{-2} \frac{\pi j}{N} \int_{-j L / N}^{j L / N} \varphi\left(\frac{2 k+j}{2 N}+\frac{t}{2 L}\right) \sin \frac{\pi t}{L} d t \\
= & \lim _{N \rightarrow \infty}\left(\pi^{3} / 2\right) N^{-2} L^{-1} \sum_{j=1}^{N-1} \sin ^{-2} \frac{\pi j}{N} \\
& \quad \int_{-j L / N}\left[\int_{0}^{N} \varphi\left(\frac{2 k+j}{2 N}+\frac{t}{2 L}\right) d k\right] \sin \frac{\pi t}{L} d t
\end{aligned}
$$

as the difference between the sum over $k$ and the respective definite integral decays exponentially with $N$ thanks to the periodicity of the integrand [22]. Since

$$
\int_{0}^{N} \varphi\left(\frac{2 k+j}{2 N}+\frac{t}{2 L}\right) d k=\int_{0}^{N} \varphi\left(\frac{k}{N}\right) d k
$$

due to the periodicity of $\varphi(t)$ [compare Eq. (6)], the first-order contribution vanishes.

The second-order contribution reads

$$
\begin{aligned}
C_{\Xi}^{(2)}= & \lim _{N \rightarrow \infty} \sum_{j=1}^{N-1}\left\{\left(\pi^{4} / 2\right) N^{-2} L^{-1} \sin ^{-2} \frac{\pi j}{N} \int_{0}^{N} d k\right. \\
& \times \int_{-j L / N}^{j L / N}\left[\varphi\left(\frac{2 k+j}{2 N}+\frac{t}{2 L}\right)\right]^{2} \cos \frac{\pi t}{L} d t+\left(\pi^{5} / 4\right) N^{-2} L^{-2} \sin ^{-3} \frac{\pi j}{N} \\
& \times \int_{0}^{N} d k\left(2\left[\int_{-j L / N} \varphi\left(\frac{2 k+j}{2 N}+\frac{t}{2 L}\right) \sin \frac{\pi t}{L} d t\right]^{2}\right. \\
& \left.\left.\left.-\int_{-j L / N}^{j L}\right]^{2}\right)\right\} \\
= & \left.\lim _{N \rightarrow \infty} \sum_{j=1}^{N-1}\left\{\left(\frac{2 k+j}{2 N}+\frac{t}{2 L}\right) \cos \frac{\pi t}{L} d t\right] \pi^{4} / 2\right) N^{-2} L^{-1} \sin ^{-2} \frac{\pi j}{N}
\end{aligned}
$$




$$
\begin{aligned}
\times & \int_{-j L / N}^{j L / N}\left(\int_{0}^{N}\left[\varphi\left(\frac{2 k+j}{2 N}+\frac{t}{2 L}\right)\right]^{2} d k\right) \cos \frac{\pi t}{L} d t \\
+ & \left(\pi^{5} / 4\right) N^{-2} L^{-2} \sin ^{-3} \frac{\pi j}{N} \\
& \times \int_{-j L / N-j L / N}^{j L / N} \int_{0}^{j L / N}\left[\int_{j}^{N} \varphi\left(\frac{2 k+j}{2 N}+\frac{t}{2 L}\right) \varphi\left(\frac{2 k+j}{2 N}+\frac{t^{\prime}}{2 L}\right) d k\right] \\
& \left.\times\left(2 \sin \frac{\pi t}{L} \sin \frac{\pi t^{\prime}}{L}-\cos \frac{\pi t}{L} \cos \frac{\pi t^{\prime}}{L}\right) d t d t^{\prime}\right\} \\
= & \lim _{N \rightarrow \infty} N^{-1} \sum_{j=1}^{N-1}\left\{\pi^{3} \sin ^{-1} \frac{\pi j}{N} \int_{0}^{1}[\varphi(\kappa)]^{2} d \kappa\right. \\
& +\left(\pi^{5} / 4\right) \sin { }^{-3} \frac{\pi j}{N} \int_{-j / N-j / N}^{j / N}\left[\int_{0}^{1} \varphi\left(k-\frac{\tau^{\prime}-\tau}{4}\right) \varphi\left(\kappa+\frac{\tau^{\prime}-\tau}{4}\right) d \kappa\right] \\
& \left.\times\left(2 \sin \pi \tau \sin \pi \tau^{\prime}-\cos \pi \tau \cos \pi \tau^{\prime}\right) d \tau d \tau^{\prime}\right\},
\end{aligned}
$$

where the periodicity of $\varphi(t)$ has been exploited again. Further simplification of the above expression yields

$$
\begin{aligned}
C_{\Xi}^{(2)}= & \lim _{N \rightarrow \infty}\left(\pi^{5} / 4\right) N^{-1} \sum_{j=1}^{N-1} \sin ^{-3} \frac{\pi j}{N} \\
& \times \int_{-j / N}^{j / N} \int_{-j / N}^{j / N}\left(2 \sin \pi \tau \sin \pi \tau^{\prime}-\cos \pi \tau \cos \pi \tau^{\prime}\right) \\
& \times\left(\int_{0}^{1}\left[\varphi\left(\kappa-\frac{\tau^{\prime}-\tau}{4}\right) \varphi\left(\kappa+\frac{\tau^{\prime}-\tau}{4}\right)-[\varphi(\kappa)]^{2}\right] d \kappa\right) d \tau d \tau^{\prime} \\
= & \lim _{N \rightarrow \infty}\left(\pi^{5} / 4\right) N^{-1} \sum_{j=1}^{N-1} \sin ^{-3} \frac{\pi j}{N} \\
& \times \int^{2 j / N}\left(\int_{0}^{1}\left[\varphi\left(\kappa-\frac{\xi}{4}\right) \varphi\left(\kappa+\frac{\xi}{4}\right)-[\varphi(\kappa)]^{2}\right] d \kappa\right) \\
& \quad-2 j / N) \\
& \quad\left[\left(\frac{j}{N}-\frac{|\xi|}{2}\right) \cos \pi \xi-(3 / 2) \pi^{-1} \sin \pi\left(\frac{2 j}{N}-|\xi|\right)\right] d \xi
\end{aligned}
$$




$$
\begin{gathered}
=\left(\pi^{5} / 2\right) \int_{0}^{1} \int_{0}^{1} \int_{0}^{1}\left[\varphi\left(\kappa-\frac{\zeta \xi}{2}\right) \varphi\left(\kappa+\frac{\zeta \xi}{2}\right)-[\varphi(\kappa)]^{2}\right] \zeta^{2} \sin ^{-3} \pi \zeta \\
\quad \times\left[2(1-\xi) \cos 2 \pi \zeta \xi-(3 / \pi) \zeta^{-1} \sin 2 \pi \zeta(1-\xi)\right] d \kappa d \xi d \zeta .
\end{gathered}
$$

Additional insights into properties of $C_{\Xi}^{(2)}$ are gained from the Fourier representation of $\varphi(t)$ [note the absence of the terms with $p=1$; compare the comment following Eq. (7)]

$$
\varphi(t)=A_{0}+\sum_{p=2}^{\infty}\left(A_{p} \cos 2 \pi p t+B_{p} \sin 2 \pi p t\right)
$$

which, when inserted into Eq. (19), produces

$$
\begin{aligned}
C_{\mathrm{\Xi}}^{(2)}= & \left(\pi^{2} / 4\right) \sum_{p=2}^{\infty} p^{2}\left(p^{2}-1\right)^{-2} \\
& \times\left[\left(8 p^{2}-6\right) H_{2 p}-\left(4 p^{2}-3\right) H_{p}-\left(6 p^{2}-4\right)\right]\left(A_{p}^{2}+B_{p}^{2}\right) \geq 0,
\end{aligned}
$$

where $H_{p}$ is the $p$-th harmonic number. Thus, as expected, $C_{\Xi}^{(2)}$ is non-negative.

In the above considerations, the relaxation of the positions of charges from those given by the minimizer (11) has not been taken into account. In order to estimate the lowering of the electrostatic self-energy due to this relaxation, one has to compute the respective leading asymptotic contributions to the energy gradient $\mathbf{g}(N)=\left\{g_{k}(N)\right\}$ and the Hessian $\mathbf{H}(N)=\left\{H_{k l}(N)\right\}$. After some algebra one obtains

$$
\begin{aligned}
g_{k}(N)=2 \pi^{3} L^{-2}\left(\sum_{j=1}^{N-1} \sin ^{-1} \frac{\pi j}{N}\right) \varphi\left(\frac{k}{N}\right)+\left(\pi^{4} / 2\right) L^{-3} \sum_{j=1}^{N-1} \sin ^{-3} \frac{\pi j}{N} \\
\quad \times \int_{-j L / N}^{j L / N} \varphi\left(\frac{j+2 k}{2 N}+\frac{t}{2 L}\right)\left[3 \sin \left(\frac{\pi t}{L}-\frac{\pi j}{N}\right)+\sin \left(\frac{\pi t}{L}+\frac{\pi j}{N}\right)\right] d t
\end{aligned}
$$

and

$$
H_{k l}(N)=N^{-1} \sum_{p=1}^{N} \eta_{p}(N) \cos \frac{2 \pi p(k-l)}{N},
$$


where

$$
\begin{aligned}
\eta_{p}(N) & =\eta_{N-p}(N)=2 \pi^{3} L^{-3} \sum_{j=1}^{N-1}\left(2 \sin ^{-3} \frac{\pi j}{N}-\sin ^{-1} \frac{\pi j}{N}\right) \sin ^{2} \frac{\pi p j}{N} \\
& =2 \pi^{3} L^{-3}\left[2 p^{2} \sum_{j=1}^{N-1} \sin ^{-1} \frac{\pi j}{N}-\sum_{q=1}^{p}(2 p-2 q+1)^{2} \cot \frac{\pi}{2 N}(2 q-1)\right] .
\end{aligned}
$$

Consequently, the leading term in the relaxation contribution to the electrostatic selfenergy equals

$$
\begin{aligned}
\Delta E_{\Xi}(N)= & -(1 / 2) \sum_{k=1}^{N} \sum_{l=1}^{N}[H(N)]_{k l}^{-1} g_{k}(N) g_{l}(N) \\
= & -(1 / 2) N^{-1} \sum_{p=1}^{N-1} \eta_{p}(N)^{-1} \sum_{k=1}^{N} \sum_{l=1}^{N} \cos \frac{2 \pi p(k-l)}{N} g_{k}(N) g_{l}(N) \\
= & -N \sum_{p=1}^{N-1}\left[\eta_{p}(N)\right]^{-1}\left(\left[\gamma_{p}(N)\right]^{2}\left(A_{p}^{2}+B_{p}^{2}\right)\right. \\
& \left.+\gamma_{p}(N) \gamma_{N-p}(N)\left(A_{p} A_{N-p}-B_{p} B_{N-p}\right)\right)
\end{aligned}
$$

where

$$
\gamma_{p}(N)=\pi^{3}\left(p^{2}-1\right)^{-1} L^{-2} \sum_{q=1}^{p}(p+1-2 q)(2 p+1-2 q) \cot \frac{\pi}{2 N}(2 q-1) .
$$

In Eq. (25), $[\mathbf{H}(N)]^{-1}$ denotes the generalized inverse of $\mathbf{H}(N)$. The identity

$$
\begin{aligned}
& \sum_{j=1}^{N-1} \sin ^{-3} \frac{\pi j}{N} \sin ^{2} \frac{\pi p j}{N} \\
& \quad=p^{2} \sum_{j=1}^{N-1} \sin ^{-1} \frac{\pi j}{N}-2 \sum_{q=1}^{p-1}(p-q)(p+1-q) \cot \frac{\pi}{2 N}(2 q-1)
\end{aligned}
$$

has been employed in the derivation of Eq. (26).

Since for large $N$ the ratios $\left\{\gamma_{p}(N) / N\right\}$ tend to finite constants whereas $\eta_{p}(N)$ grows like $N \ln N$ [21], the leading term in the large- $N$ asymptotics of $\Delta E_{\Xi}(N)$ is proportional to $N^{2}(\ln N)^{-1}$. Consequently, the geometry relaxation does not contribute to the characteristic energy. 


\section{An example}

The validity of the expressions derived in the preceding section of this paper is readily illustrated using a family of test curves of unit length with

$$
\varphi(t)=(a / 4 \pi) \sin 4 \pi t
$$

as an example. The curves $\Xi \equiv\{I(a, s),-I(-a,(1 / 4)-s)\}$, where

$$
I(a, s)=\int_{0}^{s} \cos [2 \pi t+(a / 2) \sin 4 \pi t] d t,
$$

are simple for $|a|<a_{\text {crit }} \approx 2.34213$. Their shapes change gradually with increasing $|a|$ from circular to ovoid and ladyfinger-like (Fig. 1); the curves with $a$ and $-a$ being related by permutation of the $x$ and $y$ axes. Application of the Jacobi-Anger expansion [23] allows for rapid computation of $I(a, s)$ as

$$
\begin{aligned}
I(a, s)= & J_{0}(a / 2) \frac{\sin 2 \pi s}{2 \pi} \\
& +\sum_{n=1}^{\infty} J_{n}(a / 2)\left[(-1)^{n} \frac{\sin 2 \pi(2 n-1) s}{2 \pi(2 n-1)}+\frac{\sin 2 \pi(2 n+1) s}{2 \pi(2 n+1)}\right],
\end{aligned}
$$

a

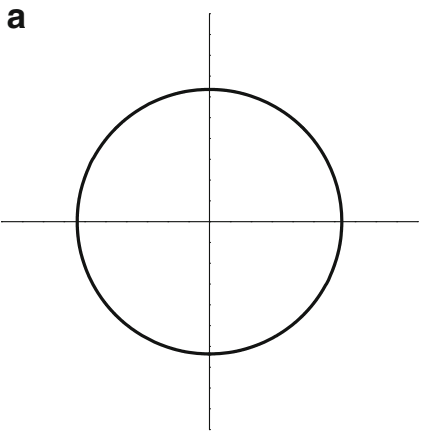

C

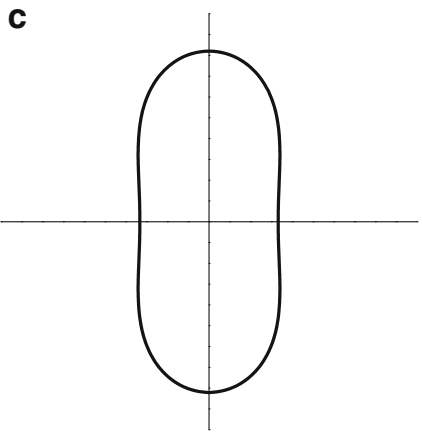

b

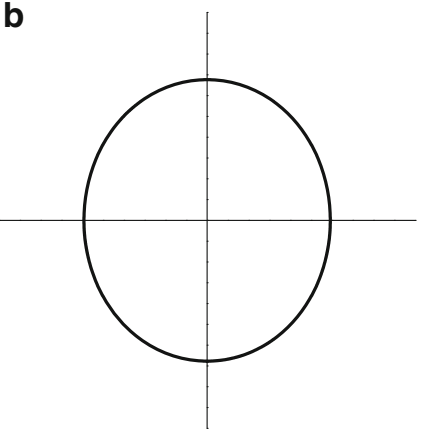

d

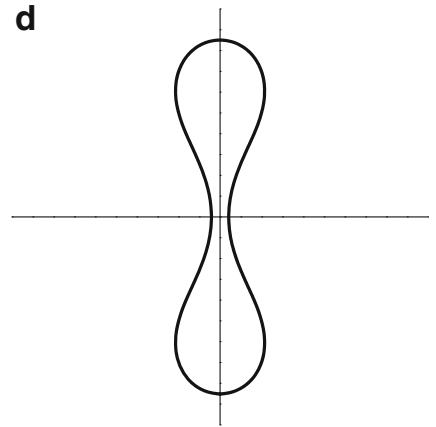

Fig. 1 The test curve for $\mathbf{a} a=0, \mathbf{b} a=0.2$, c $a=1.2$, and $\mathbf{d} a=2.2$ 
Table 1 The values of $a^{-2} N^{-2}\left[E_{\Xi}(N)-E_{\text {circle }}(N)\right]$ for a family of the test curves [Eq. (28)]

\begin{tabular}{llllll}
\hline $\mathrm{N}$ & \multicolumn{5}{c}{$\mathrm{a}$} \\
\cline { 2 - 6 } & 0.001 & 0.01 & 0.1 & 1.0 & 2.0 \\
\hline 10 & 0.1011676491 & 0.1011683902 & 0.1012425763 & 0.1094965415 & 0.1566486509 \\
100 & 0.1018450054 & 0.1018457466 & 0.1019199271 & 0.1101737723 & 0.1556763618 \\
1,000 & 0.1018517909 & 0.1018525319 & 0.1019267124 & 0.1101805575 & 0.1556831468 \\
10,000 & 0.1018518589 & 0.1018525997 & 0.1019267802 & 0.1101806253 & 0.1556832146 \\
100,000 & 0.1018518598 & 0.1018526004 & 0.1019267809 & 0.1101806260 & 0.1556832153 \\
\hline
\end{tabular}

where $J_{n}(t)$ is the Bessel function of the first kind. In practice, sufficient numerical accuracy is attained with the first 100 terms of the expansion (30), the Bessel functions being evaluated with Miller's algorithm [23,24].

Substitution of Eq. (28) into either Eq. (19) or Eq. (21) yields $C_{\Xi}^{(2)}=(11 / 108) a^{2} \approx$ $0.1018518519 a^{2}$. Inspection of Table 1 , in which the scaled excess energies $a^{-2} N^{-2}\left[E_{\Xi}(N)-E_{\text {circle }}(N)\right]$ for sets of $N$ charges located at the values of $\left\{s_{k}\right\}$ equal to multiples of $N^{-1}$ are compiled, reveals several facts. First of all, the unscaled excess energies are indeed asymptotically proportional to squares of the particle numbers. Second, for sufficiently small values of $a$, they are proportional to $a^{2}$. Third, the value of $C_{\Xi}^{(2)}$ extracted from numerical calculations coincides with the pertinent theoretical prediction.

Comparison of the predicted relaxation contributions to the electrostatic self-energy with their numerical counterparts is equally instructive. According to Eqs. (24)-(26),

$$
\begin{aligned}
\Delta E_{\Xi}(N)=- & (\pi / 288) a^{2} N\left(3 \cot \frac{\pi}{2 N}-\cot \frac{3 \pi}{2 N}\right)^{2} \\
& \times\left(8 \sum_{j=1}^{N-1} \sin ^{-1} \frac{\pi j}{N}-9 \cot \frac{\pi}{2 N}-\cot \frac{3 \pi}{2 N}\right)^{-1} \\
=- & \left(a^{2} / 162\right)\left(N^{2} / \ln N\right)+\cdots .
\end{aligned}
$$

\begin{tabular}{|c|c|c|c|c|c|c|}
\hline \multirow[t]{2}{*}{$\mathrm{N}$} & \multicolumn{6}{|c|}{$\mathrm{a}$} \\
\hline & $0^{\mathrm{a}}$ & 0.001 & 0.01 & 0.1 & 1.0 & 2.0 \\
\hline 10 & -0.0111718091 & -0.0111718137 & -0.0111722341 & -0.0112143970 & -0.0164464157 & -0.0680969401 \\
\hline 100 & -0.0079755931 & -0.0079755946 & -0.0079759090 & -0.0080072518 & -0.0119730987 & -0.0543049725 \\
\hline 1,000 & -0.0072681916 & -0.0072681914 & -0.0072684819 & -0.0072972827 & -0.0109564209 & -0.0512516844 \\
\hline 10,000 & -0.0069594588 & -0.0069594609 & -0.0069597377 & -0.0069874137 & -0.0105099478 & -0.0498666997 \\
\hline 100,000 & -0.0067864950 & -0.0067864993 & -0.0067867675 & -0.0068138095 & -0.0102590850 & -0.0490751108 \\
\hline
\end{tabular}

Table 2 The values of $a^{-2} N^{-2} \ln N \Delta E_{\Xi}(N)$ for a family of the test curves [Eq. (28)]

${ }^{\text {a }}$ See Eq. (31). The data derived from the actual values of $\Delta E_{\Xi}(N)$ are listed for the non-vanishing $a$ 
The scaled relaxation energies $a^{-2} N^{-2} \ln N \Delta E_{\Xi}(N)$ listed in Table 2 closely follow this prediction for small values of $a$ although their convergence to the limiting value of $-1 / 162 \approx-0.006172839506$ is rather slow.

\section{Conclusions}

The characteristic energy $C_{\Xi}$ of a smooth Jordan curve $\Xi$ of length $L$, defined as the coefficient in the term proportional to $N^{2} / L$ in the large- $N$ asymptotics of the minimal electrostatic self-energy of $N$ unit charges located on the curve in question, possesses an expansion involving the function $\varphi(t)$ that measures the deviation from linearity in the dependence of the tangential angle on the arc length. The leading term in this expansion is given by a positive-valued functional that is quadratic in $\varphi(t)$. Consequently, at least in the local sense, a circle has the smallest $C_{\Xi}$ among all smooth Jordan curves.

The explicit expression for this second-order functional can be derived without taking into account the energy lowering due to relaxation of the particle positions that, being of the order of $N^{2}(\ln N)^{-1}$ for large $N$, does not contribute to the characteristic energy. However, in light of its complexity, the prospects for derivation of a nonperturbative closed-form expression for $C_{\Xi}$ remain remote.

Acknowledgments The research described in this publication has been funded by NCN (Poland) under Grant No. DEC-2012/07/B/ST4/00553.

Open Access This article is distributed under the terms of the Creative Commons Attribution License which permits any use, distribution, and reproduction in any medium, provided the original author(s) and the source are credited.

\section{References}

1. Y.H. Liu, L.Y. Chew, M.Y. Yu, Phys. Rev. E 78, 066405 (2008)

2. A.V. Filinov, M. Bonitz, E. Lozovik, Phys. Rev. Lett. 86, 3851 (2001)

3. B. Partoens, F.M. Peeters, J. Phys. Condens. Matter 9, 5383 (1997)

4. C. Yannouleas, U. Landman, Rep. Prog. Phys. 70, 2067 (2007)

5. S.M. Reimann, M. Manninen, Rev. Mod. Phys. 74, 1283 (2002)

6. T. Paananen, R. Egger, H. Siedentop, Phys. Rev. B 83, 085409 (2011)

7. K. Nelissen, A. Matulis, B. Partoens, M. Kong, F.M. Peeters, Phys. Rev. E 73, 016607 (2006)

8. Y-J. Lai, L. I, Phys. Rev. E 60, 4743 (1999)

9. E. Yurtsever, F. Calvo, Mol. Phys. 106, 289 (2008)

10. P. Cheung, M.F. Choi, P.M. Hui, Solid State Commun. 103, 357 (1997)

11. B.A. Grzybowski, J.A. Wiles, G.M. Whitesides, Phys. Rev. Lett. 90, 083903 (2003)

12. B.J. Baelus, L.R.E. Cabral, F.M. Peeters, Phys. Rev. B 69, 064506 (2004)

13. L.R.E. Cabral, B.J. Baelus, F.M. Peeters, Phys. Rev. B 70, 144523 (2004)

14. V.A. Schweigert, F.M. Peeters, Phys. Rev. B 51, 7700 (1995)

15. V.M. Bedanov, F.M. Peeters, Phys. Rev. B 49, 2667 (1994)

16. J. Cioslowski, J. Albin, J. Chem. Phys. 136, 114306 (2012)

17. J. Cioslowski, J. Albin, J. Chem. Phys. 139, 114109 (2013)

18. A. Martínez-Finkelshtein, V.V. Maymeskul, E.A. Rakhmanov, E.B. Saff, Canad. J. Math. 56, 529 (2004)

19. W. Whewell, Of the intrinsic equation of a curve, and its application. Camb. Philos. Trans. VIII, 659-671 (1849) 
20. J. Arroyo, O.J. Garay, J.J. Menciá, Am. Math. Monthly 115, 405 (2008)

21. J. Cioslowski, J. Albin, J. Math. Chem. 50, 1378 (2012)

22. M. Javed, L.N. Trefethen, Proc. R. Soc. A 470, 20130571 (2014)

23. M. Abramowitz, I.A. Stegun (eds.), Handbook of Mathematical Functions with Formulas, Graphs, and Mathematical Tables (Dover, New York, 1965)

24. F.S. Acton, Numerical Methods That Work (Mathematical Association of America, Washington, 1990) 